\title{
Beats + Sample Manipulation in Pure Data with Raspberry Pi
}

\author{
Nick Demopoulos
}

USA

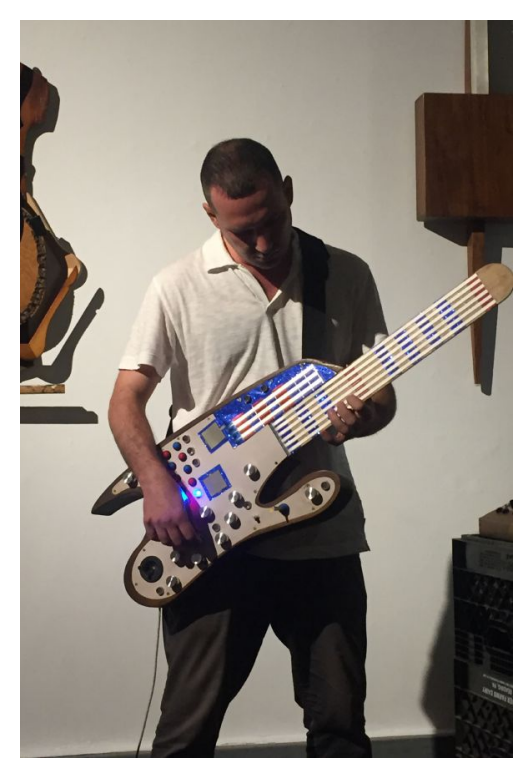

Nick Demopoulos Nick Demopoulos is a musician and artist who performs under the name Smomid. He utilizes interfaces he designed to create interactive audio-visual performances. As a guitarist Nick worked with NEA jazz master Chico Hamilton and recorded on the albums The Inquiring Mind, Revelation and Euphoric. Nick has worked for U.S. State Department and Jazz at Lincoln Center as a cultural diplomat, performing in Bahrain, Yemen, Oman, U.A.E and Kuwait. Nick has performed at Lincoln Center, Phoenix Festival, LACMA, and been featured on the Discovery Science Network, Guitar World, Create Digital Music, BOMB magazine among other media outlets.

E-mail: $\underline{\text { Smomid27@gmail.com }}$

Website: https://smomid.com/ 
$\mathrm{I}$

composed and performed the piece in the video accompanying this writing, which I call Cosmic Space ${ }^{1}$. It was performed using a MIDI controller I created called a Pyramidi that is connected to a Raspberry Pi 4 computer, with 8 gigs of RAM, that is running Pure Data. The MIDI controller is the White box with various sensors and LED displays in it. The box next to it, wrapped in copper sheeting, is the enclosure for the Raspberry Pi. For me the most significant achievement in this work was simply creating and assembling the physical hardware to facilitate music performance. The Raspberry Pi can be turned on with a button press, runs "headless," is battery powered, and is connected to a sound card. Pure Data patches are opened using the buttons on the front of the box and stored on a USB stick. The Pi automatically connects to any MIDI controller plugged into its USB port. The MIDI controller has a variety of sensors including FSR (force sensing resistor), joysticks, buttons, and Rotary potentiometers. This controller is powered by an Arduino Mega that maps electronic resistance from the sensors to MIDI control change data.

I created the drum sequencer used in this performance out of Pure Data toggle boxes. The various patterns are stored as text files. One invaluable resource for me when creating performances like this are contributions of Pure Data users that are freely available. Some of the audio effects I used in this performance include compress $\sim$ from the Else library, degrade from the Cyclone library, and both the Granular and Stereo delay from the Automatonism library. Percussion abstractions I used include membrane and cmbcyn , created by Miguel Moreno. The growing number of available effects, abstractions and libraries is making Pure Data more user friendly and easier to produce a wide variety of sounds. This aspect of Pure Data has made it favorable to me over other methods of making music. Additionally, with Pure Data being free/open source software and the Raspberry Pi 4 being \$75, this method of music performance is very accessible financially. The time and effort I invested in creating this system was considerable, but hopefully going forward I can make smaller modifications an improvements to build on the current method I now utilize.

\footnotetext{
${ }^{1}$ Full video at: https://youtu.be/YRicNKd7NJA
} 